\title{
Long-term Patterns of Patient Portal Use for Pediatric Patients at an Academic Medical Center
}

Bryan D. Steitz; Robert M. Cronin 1,2,3; Sharon E. Davis; Ellen Yan4; Gretchen P. Jackson 1,3,5

${ }^{1}$ Department of Biomedical Informatics, Vanderbilt University Medical Center; ${ }^{2}$ Department of Medicine, Vanderbilt University Medical Center; ${ }^{3}$ Department of Pediatrics, Vanderbilt University Medical Center; ${ }^{4}$ Health IT @VUMC, Vanderbilt University

Medical Center; ${ }^{5}$ Department of Pediatric Surgery, Vanderbilt University Medical Center

\section{Keywords}

Patient portal, Patient engagement, Consumer health informatics, Pediatrics, Electronic health records and systems, Internet Portal

\section{Summary}

Background: Patient portal adoption has increased over the last two decades. Most research about patient portals has focused on adult populations in the primary care and medical specialty settings. Objective: We describe initial and long-term portal use by pediatric patients and their caregivers in a broadly deployed patient portal at an academic medical center.

Methods: We analyzed portal usage for pediatric patients and their caregivers from 2008 to 2014. We recorded usage events with time stamps; user role defined as self, surrogate (i.e., parent or guardian), or delegate; and functions accessed. Usage events were grouped into sessions to calculate descriptive statistics by patient age, user role, and active use over time.

Results: From 2008 to 2014, the number of portal accounts increased from 633 to 17,128. 15.9\% of pediatric patients had their own account; $93.6 \%$, a surrogate account; and $2.2 \%$ a delegate account. During the study period, 15,711 unique users initiated 493,753 sessions and accessed $1,491,237$ functions. Most commonly used functions were secure messaging (accessed in 309,204 sessions; $62.6 \%)$; test results $(174,239 ; 35.3 \%)$ and appointments $(104,830 ; 21.2 \%)$. Function usage was greatest for patients ages $0-2$ years $(136,245$ functions accessed; $23.1 \%)$ and $15-17$ years $(109,241 ; 18.5 \%)$. Surrogate users conducted $83.2 \%$ of logins for adolescent patients. Portal accounts were actively used for $<1$ year for 9,551 patients $(55.8 \%), 1-2$ years for 2,826 patients (16.5\%), 2-3 years for 1,968 patients (11.5\%) and over 3 years for 2,783 patients (16.3\%). Conclusion: Pediatric patients and caregivers have avidly used messaging, test result, and appointment functions. The majority of access was done by surrogates. Adolescent portal usage increased with age. Most accounts for pediatric patients were only used actively for a few years, with peak usage for patients in early childhood and late adolescence.

\section{Correspondence to:}

Bryan Steitz

2525 West End Avenue

Suite 1475

Nashville, TN 37203

Tel. (502) 320-6797

Email: bryan.steitz@vanderbilt.edu
Appl Clin Inform 2017; 8: 779-793

received: January 17, 2017

accepted in revised form: May 18, 2017

published: August 2, 2017

Citation: Steitz BD, Cronin RM, Davis SE, Yan Z, Jack-

son GP. Long-term Patterns of Patient Portal Use for

Pediatric Patients at an Academic Medical Center. Appl

Clin Inform 2017; 8: 779-793

https://doi.org/10.4338/ACI-2017-01-RA-0005 


\section{Background and Significance}

Patient portals are web-based tools that allow healthcare consumers to interact with healthcare systems and health information [1-8]. Such portals commonly support the ability to view medical records, pay medical bills, securely message providers, and schedule appointments [9]. Patient portals have been implemented and used in diverse settings including large academic medical centers, community practices, primary care, specialty care, and the inpatient setting [10]. Programs delivered through patient portals have been shown to improve patient satisfaction; increase adherence to preventative care such as immunizations and treatment recommendations; and improve clinical outcomes for chronic diseases in the adult population [11-18].

Research on portal use has primarily focused on adult users in primary or chronic disease care [9, 19, 20]. A recent review of pediatric patient portals revealed only eleven studies [23], with the majority reporting qualitative studies on small populations assessing willingness to use patient portals, eliciting design recommendations, and conducting usability testing [24-29]. Three studies in pediatric primary care demonstrated relatively low uptake and reported usage disparities previously noted among adult populations [30-32]. Several studies have described usage by the pediatric patients with selected chronic diseases or their parents [33-34], with only one providing data about portal usage over time [35]. None of these studies have examined long-term usage of patient portals among pediatric populations.

With increasing consumer demand and regulatory pressures, more healthcare organizations are expected to adopt patient portals and deploy them widely. The objective of this study was to describe both initial and long-term patient portal use by pediatric patients and their caregivers in a broadly deployed patient portal at an academic medical center. We examined portal registration and usage rates by patient age and sociodemographic characteristics over time, distinguishing between usage by patients and caregivers.

\section{Methods}

\subsection{Study Setting}

The study was conducted at the Monroe Carell Jr. Children's Hospital at Vanderbilt University Medical Center (VUMC), a large, academic medical center located in middle Tennessee. During the study period, the children's hospital delivered increasing volumes of care, including 161,060 to 251,909 outpatient visits, 13,029 to 14,615 hospital discharges, and 46,978 to 52,254 emergency room encounters each year [36]. The research was conducted in compliance with the World Medical Association Declaration of Helsinki on Ethical Principles for Medical Research Involving Human Subjects, and the VUMC Institutional Review Board approved this study. VUMC offers an institutionally-developed patient portal called My Health at Vanderbilt (MHAV), which launched in 2005 and was deployed throughout all clinical specialties, including the initiation of pediatric accounts in 2007 [37, 38]. MHAV and its tethered electronic health record (EHR) were certified for Meaningful Use Stage 2. MHAV provides common patient portal functions including access to administrative forms, appointment scheduling, account management and bill paying, secure messaging with healthcare providers, and access to selected portions of the EHR such as test results, visit summaries, vital signs, and immunizations. Test results are displayed immediately, with a 3-day delay or with a 7-day delay depending on sensitivity of the test result. Tests that have been classified as containing highly sensitive information such as those related to certain sexually transmitted infections are not displayed through MHAV. MHAV is available to all patients receiving care at VUMC.

For pediatric patients less than 18 years of age, parents or legal guardians can access their child's health information through surrogate accounts. Delegate accounts may also be established to grant access to other caregivers, such as stepparents or chronic care nurses. With parental approval and patient assent, pediatric patients older than 13 years of age may have their own MHAV accounts, with access to all pediatric patient information available to both patients, delegates, and surrogates. Thus, for pediatric patients, MHAV usage may be conducted by self, delegate, and surrogate users. In Tennessee, the ability to control access to personal health information is linked to ability to pro- 
vide consent for treatment, which can be granted on a case-by-case basis for adolescents less than 18 years of age. Surrogate and delegate accounts remain active until the patient turns 18 years of age or is deemed able to provide consent, at which time surrogate access is terminated and delegate access is at the patient's discretion. At the early stages of portal adoption, some self accounts were inadvertently created for younger patients and were presumably used by the parents. We have included usage data for these accounts in our analysis to measure accurately function usage about patients of a particular age category. MHAV currently has over 400,000 registered users with over 300,000 logins per month by approximately 50,000 unique users. There are currently over 22,500 accounts for pediatric patients or their caregivers.

\subsection{Study Population}

We examined all access to MHAV related to pediatric patients less than 18 years of age, between August 1, 2007 and December 31, 2014. This study period supports the examination of usage patterns since the initiation of pediatric accounts. For those patients reaching 18 years of age during the study, we only analyzed usage occurring prior to their 18th birthday. We collected data on all MHAV usage events, including user role, described as self, surrogate, or delegate; a time stamp; and functions accessed. Automated or MHAV administrator account access events were excluded.

\subsection{Data Analysis}

We assigned all usage events to four categories including appointment functions, billing, messaging, and test results ( $\triangleright$ Fig. 1). Usage events were grouped by sessions. A session was defined as any sequence of patient portal usage events by a single user for a unique patient that occurred within 30 minutes after any previous use without any login or logout events. All analyses, with the exception of summary statistics, were reported in the context of sessions. Summary statistics were calculated to evaluate the number of sessions and functions accessed across age groups, demographics, and user types. We tested for linear changes in function use over time. To determine whether users remained active over time, we evaluated account activity for each twelve-month period beginning at the date of account creation. Active account use for a pediatric patient was defined as any successful login or use of MHAV by the patient or an associated delegate or surrogate. The analysis was conducted in $\mathrm{R}$ version 3.2.2 [39].

\section{Results}

In the first year of MHAV deployment for pediatric patients (2007), accounts were created for 633 pediatric patients. By 2014, 17,128 patients had an associated MHAV account ( $\$$ Fig. 2). The number of new pediatric patients enrolled in MHAV each year rose steadily from 2007 to 2012, after which the number of new accounts plateaued. Among all pediatric patients enrolled in MHAV, $2,720(15.9 \%)$ had their own account, $16,036(93.6 \%)$ had a surrogate account, and $378(2.2 \%)$ had a delegate account. Each patient was associated with a mean of 1.1 accounts. Table 1 shows the sex, race, and age at enrollment of patients with MHAV accounts. During the study period, 1406 self accounts were inadvertently created for patients less than 13 years of age, 1099 (78\%) of which were created in the first two years after deployment of pediatric accounts. It is likely that these accounts were used by parents rather than children, and thus, the results for this group are shown separately in $>$ Table 1. Patients with MHAV access were predominately Caucasian (80.1\%), with a median age of 8 years at MHAV enrollment.

During the study period, 493,753 MHAV sessions were initiated by 15,711 distinct users, with a median of 8 sessions per user. $>$ Figure 3 presents MHAV use by function type and year. Users accessed an average of 13.8 functions per year. There was a significant increase in total function usage across years $(\mathrm{p}<0.0001)$. Secure messaging was the most popular MHAV function, accessed in $309,204(62.6 \%)$ sessions. Test result and appointment functions were accessed in 174,239 (35.3\%) and 104,830 (21.2\%) sessions, respectively. Billing functions were the least frequently used, accessed in only $1,156(0.2 \%)$ sessions. Use of the test result function increased annually, with a maximum ac- 
cess in 37,140 sessions in 2014. Messaging saw a yearly increase until reaching a maximum of access in 64,826 unique sessions in 2013. Appointment and billing functions had the most use in 2014 $(28,802)$ and 2010 (358), respectively.

MHAV function usage by patient age is shown in $>$ Figure 4 . Use of all MHAV functions were greatest for pediatric patients of ages 0 to 2 years and 15 to 17 years, with $136,245(23.1 \%)$ and 109,241 (18.5\%) functions being accessed for these age groups during the study. The messaging, test result, and appointment functions were used most frequently for patients 1 year of age with 25,689 (8.3\%), 15,960 (9.2\%), and 9,175 (8.8\%) accesses, respectively. These three functions were accessed least frequently for patients of age 13 years of age, with corresponding accesses of 13,889 (4.5\%), 7,025 (4\%), and 4,226 (4\%). The billing function had the most use (112 accesses) for patients 17 years of age and the least use (39 accesses) for patients 10 years of age.

For most patients $(9,551 ; 55.8 \%)$, MHAV accounts were active for less than one year. The duration of MHAV active use for 2,826 patients (16.5\%) was 1-2 years and for 1,968 patients (11.5\%) was 2-3 years. Active MHAV use of 3-4 years was observed for 1,227 patients (7.2\%), and 1,556 patients $(9.1 \%)$ were active on MHAV for more than four years. Among patients under 18 years of age for the entire study, we observed a similar pattern, with 7,877 (54.9\%) remaining active for less than one year, 2,358 (16.4\%) for 1-2 years, 1,669 (11.6\%) for 2-3 years, 1,049 (7.3\%) for 3-4 years, and $1,400(9.8 \%)$ for more than four years.

We analyzed adolescent usage by user role, as patients older than 13 years of age were allowed to have their own accounts with parental permission. For adolescent patients, there were 1,388 self, 3,967 surrogate, and 43 delegate accounts established during the study period. Surrogate users accounted for 115,329 (83.2\%) of all logins for adolescent patients. Patient and delegate users accounted for 22,846 (16.5\%) and $513(0.3 \%)$ logins, respectively. For both patient and surrogate accounts, the majority of logins occurred for patients 16 years of age, with 6,715 (29.4\% of adolescent patient logins) and 25,584 (22.2\% of adolescent surrogate logins) corresponding successful logins. The majority of delegate account logins occurred for patients 17 years of age with 134 logins (29.2\% of adolescent delegate logins) during the study period. All three account types had the lowest number of logins for patients 13 years of age. $>$ Figure 5 presents login statistics by role.

\section{Discussion}

This study is one of the first to examine long-term trends in patient portal usage for pediatric patients. We demonstrated rapid growth in portal usage by pediatric patients and their caregivers in the initial years after deployment of pediatric accounts at a large academic medical center. At our institution, the number of registered users and usage of individual functions increased dramatically over the first several years and then plateaued, a pattern seen in adult portal studies [40]. We also observed a steady increase in total users but a relatively stable number of new enrollees each year. Such usage patterns can inform other academic pediatric medical centers that are in the early stages of portal implementation. Although many institutions report growing number of portal users, an important consideration is whether users remain active after registration. In this study, over half of pediatric accounts were active for less than one year, a finding which has important implications if ongoing patient portal engagement is a considered as a measure of Meaningful Use. This episodic usage pattern may reflect the population receiving highly specialized care at our institution and should be compared with portal usage patterns in predominantly primary care settings in future studies.

As healthcare organizations adopt online tools, the disparities in access to care created by such technologies must be considered. In our study, the majority of patients for whom portal accounts were created were Caucasian, a finding that is not surprising as the referral base for VUMC is $77-87 \%$ Caucasian $[41,42]$. Other studies have reported similar disparities with a disproportionate number of Caucasian users in both pediatric [35, 43] and adult $[4,38,40]$ populations. At our institution, pediatric accounts were created for male and female pediatric patients in nearly equal numbers. One significant limitation of our work is that we do not know the demographic characteristics of the delegate or surrogate users. Our demographic data do describe disparities in the patients receiving care through the portal, but observed disparities in healthcare access for surrogate and 
delegate users may differ. Since the majority of portal users for pediatric accounts were surrogates, we hypothesize that the majority of these users were female, as found in other studies [4, 44]. Females are reported to make $80 \%$ of healthcare decisions for families [45], and thus, it is not unexpected that women are more likely to be the ones seeking access to providers and healthcare institutions with online tools.

In the eight years after deployment of pediatric accounts, MHAV activity for pediatric accounts steadily increased. Such growth, particularly in secure messaging, may produce additional work for pediatric providers. The number of functions accessed stayed consistent at an average of nearly fourteen yearly function accesses per patient, but the number and type of functions accessed varied widely across patients and age groups. As in other adult and pediatric studies [44, 47], secure messaging was the most frequently used function accessed by pediatric accounts, with nearly twice as many uses as the test result functions. Messaging also had the fastest growth in the number of accesses during our study period. Increased provider adoption and support of messaging over time at our institution was likely an important factor in this sustained growth, as provider encouragement has been shown to be an important factor in patients' willingness to use portal messaging [44]. As portal messaging adoption increases, the effects on provider workload need to be carefully considered, and models for reimbursement of online care should be developed. Analyses of MHAV message exchanges has demonstrated that the majority involve delivery of medical care, and this substantial provider effort is uncompensated $[48,49]$.

This study is one of the first studies to explore usage of a broadly deployed patient portal for pediatric patients by age. In our study, usage of the pediatric portal accounts varied by age of the patient, with the highest usage occurring during the first three years of life and last three years of adolescence. Patient portal accounts for patients under two years of age had the most logins, which is likely due to the increased medical care required during this crucial time for infants. A study by Byczkows$\mathrm{ki}$ and colleagues noted an association between more outpatient visits and increased portal use [35]. Since MHAV portal messaging is more likely to be used by pediatric specialists than pediatric primary care providers, we cannot fully attribute our observations to the increased number of well child visits in the first years of life [50]. Our children's hospital does provide perinatal services not offered elsewhere in the region, such as an advanced maternal care center, fetal surgical interventions, and extracorporeal membrane oxygenation, so many infants with congenital anomalies are born at Vanderbilt and remain at our institution for care early in life. This population likely contributes significantly to the increased usage of portal functions for patients in the first years of life.

Our observation of increased usage for patients in late adolescence could be related to the transition period from childhood into adulthood, a vulnerable time for many pediatric patients and their parents, especially those with chronic diseases. The American Academy of Pediatrics (AAP) developed an algorithm that urges providers to improve the transition period by encouraging adolescents to take a more active role in disease self-management and health maintenance through increased interaction with the healthcare system [51]. Few studies have explored independent use of patient portals by adolescent patients [30-32]. Although adolescent patients are eligible for their own MHAV accounts at VUMC, over $83 \%$ of the logins for these patients were by done by surrogate users. In contrast, another study of a patient portal specifically designed for adolescent health care needs in the primary care setting saw frequent access by adolescent users [30]. In our study, the number of "self" logins for teenage users increased with the patient age, which could be attributed to adolescent patients becoming more involved in their care as they mature, being promoted by providers as recommended by the AAP, or becoming simply more familiar with using MHAV. The number of surrogate logins for adolescent patients also increased with patient age, suggesting that parents and other caregivers remain intimately involved in the patient's care. Differences in adolescent usage can be attributed in part to the institutional privacy and confidentiality policies. At VUMC, our approach favors parental access to a children's information whereas portals that prioritize adolescent confidentiality, allowing parents only limited information, show higher rates of adolescent usage [30]. One organization that required adolescent assent for proxy access showed low rates of registration for such accounts, suggesting that adolescents may have reservations about sharing their health data with their parents [32]. Laws that govern privacy of health information for adolescents vary widely from state to state, and these regulations must be taken into account in developing policies for adolescent patient portal access. 
This study has several limitations. First our data comes from a single, large academic medical center with a locally-developed patient portal, which may limit the generalizability of the findings. However, MHAV has core functions similar to those of most patient portals, and this study demonstrates long-term usage trends of the most common portal functions. Additionally, this study only examined patient portal activity by patient age, demographic characteristics, and user role. We did not have patient-specific clinical data for the pedatric MHAV accounts, and thus did not analyze other clinical factors that might affect usage such as patient diagnoses, insurance status, healthcare provider usage, or healthcare utilization factors, such as office visits, telephone calls, or hospital admissions. In addition, our demographic data only included patient demographics, not the characteristics of the surrogate or delegate users. Our ongoing research is exploring patient portal usage across clinical specialties and the relationships across portal usage, clinical variables, and user variables, such as health literacy or patient activation.

\section{Conclusion}

To our knowledge, this study is one of the first to characterize comprehensively long-term patterns of patient portal use for pediatric patients. We have demonstrated that pediatric patients and their caregivers embrace patient portals through avid use of messaging, viewing test results, and appointment scheduling functions. At our institution, caregivers for patients less than two years of age were the most active patient portal users, and surrogate users (i.e., parents or guardians) most frequently accessed pediatric information through the portal for all ages, a trend most likely due to institutional policies and state laws that favor parental rights over adolescent privacy. Some adolescent pediatric patients used patient portals to interact with the healthcare system on their own, with older adolescents using the portal more frequently. Long-term active portal usage was uncommon as most patients and caregivers used the portal for a year or less. As patient portal adoption increases for children in response to consumer demand and regulatory requirements, this study provides guidance for long-term usage trends. Additional research is needed to understand the relationships between patient portal usage for pediatric patients and patient and family activation, self-efficacy, and clinical outcomes.

\section{Clinical Relevance Statement}

Patient portals have been shown to increase patient satisfaction, enhance communication, and improve outcomes. Most studies of patient portal usage by pediatric patients and their caregivers have examined initial adoption, usually in primary care settings or patients with chronic diseases. This study is the first to evaluate comprehensively the long-term patient portal usage trends for pediatric patients at an academic medical center.

\section{Multiple Choice Questions}

Adolescent access to health information through patient portals is:

a. Required after age 13 years

b. Allowed with parent permission after age 13 years

c. Determined by state laws, which vary widely

d. Forbidden until age 18 years

The correct answer is c. Laws that govern privacy of health information for adolescents vary widely from state to state. These regulations must be taken into account in developing policies for adolescent patient portal access.

\section{Conflicts of Interest}

The authors have no conflicts of interest relevant to this article to disclose. 


\section{Protection of Human and Animal Subjects}

The VUMC IRB approved this study. The study was performed in compliance with the World Medical Association Declaration of Helsinki on Ethical Principles for Medical Research Involving Human Subjects. Waiver of consent was granted by the IRB for this study involving no more than minimal risk and not adversely affecting the rights and the welfare of relevant individuals.

\section{Acknowledgements}

Bryan Steitz and Sharon Davis were supported by the 4T15LM007450 training grant from the National Library of Medicine. 


Appointment Functions
User Viewed Appointments
View Appointments
Request Appointment New Doc
Billing Functions
View Billing
View Insurance
Billing Question
Bill Payment Entry
Bill Payment Completion
Messaging Functions
View Secure Messaging
Message - Delete
Message - Forward
Message - Reply
Message - Send New
Message - View
Test Result Functions
View Labs Screen

Fig. 1 MHAV Functions 


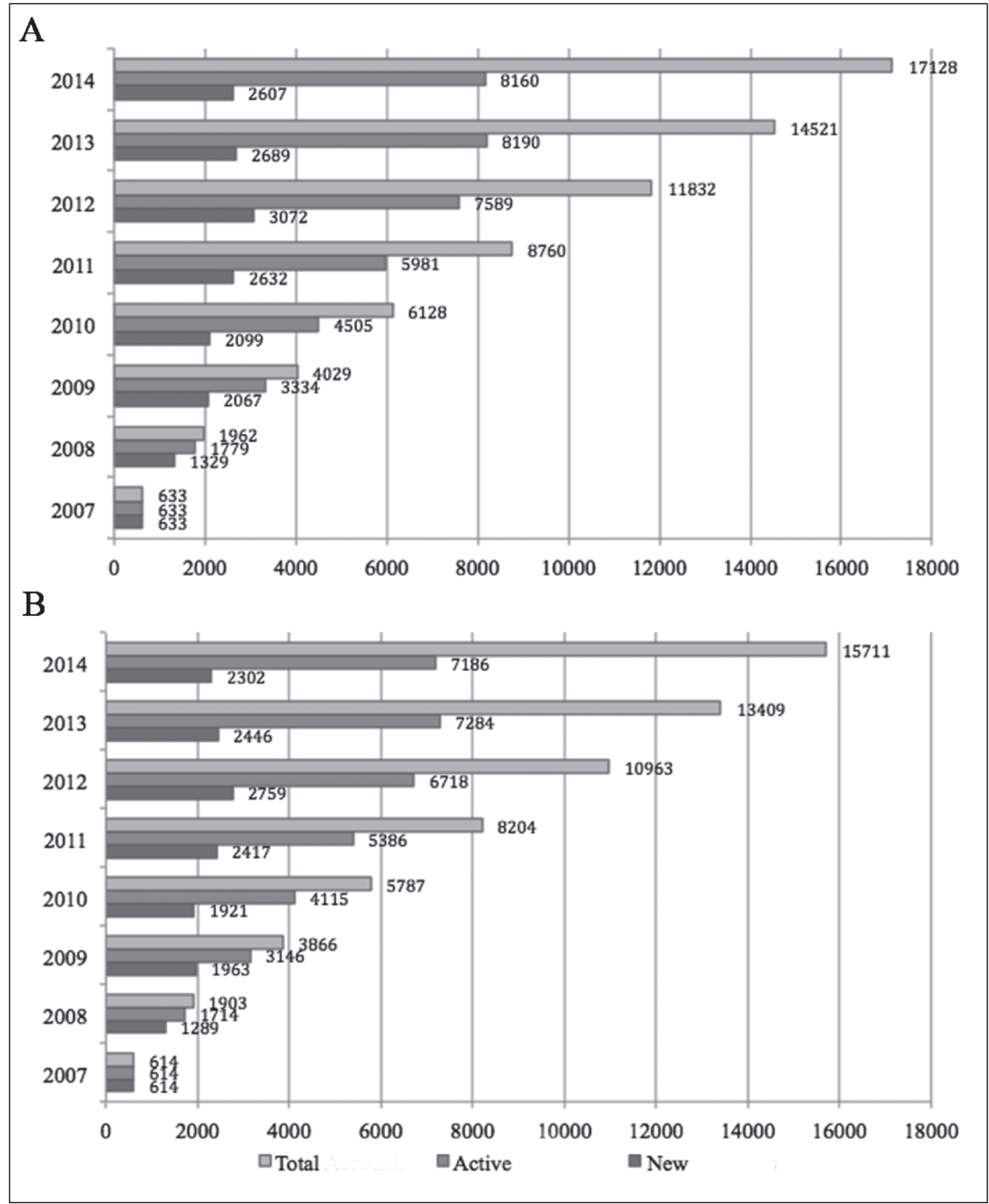

Fig. 2 A) Number of total, active and new pediatric patients with MHAV by year, and B) Number of total and new MHAV accounts for pediatric patients by year. 


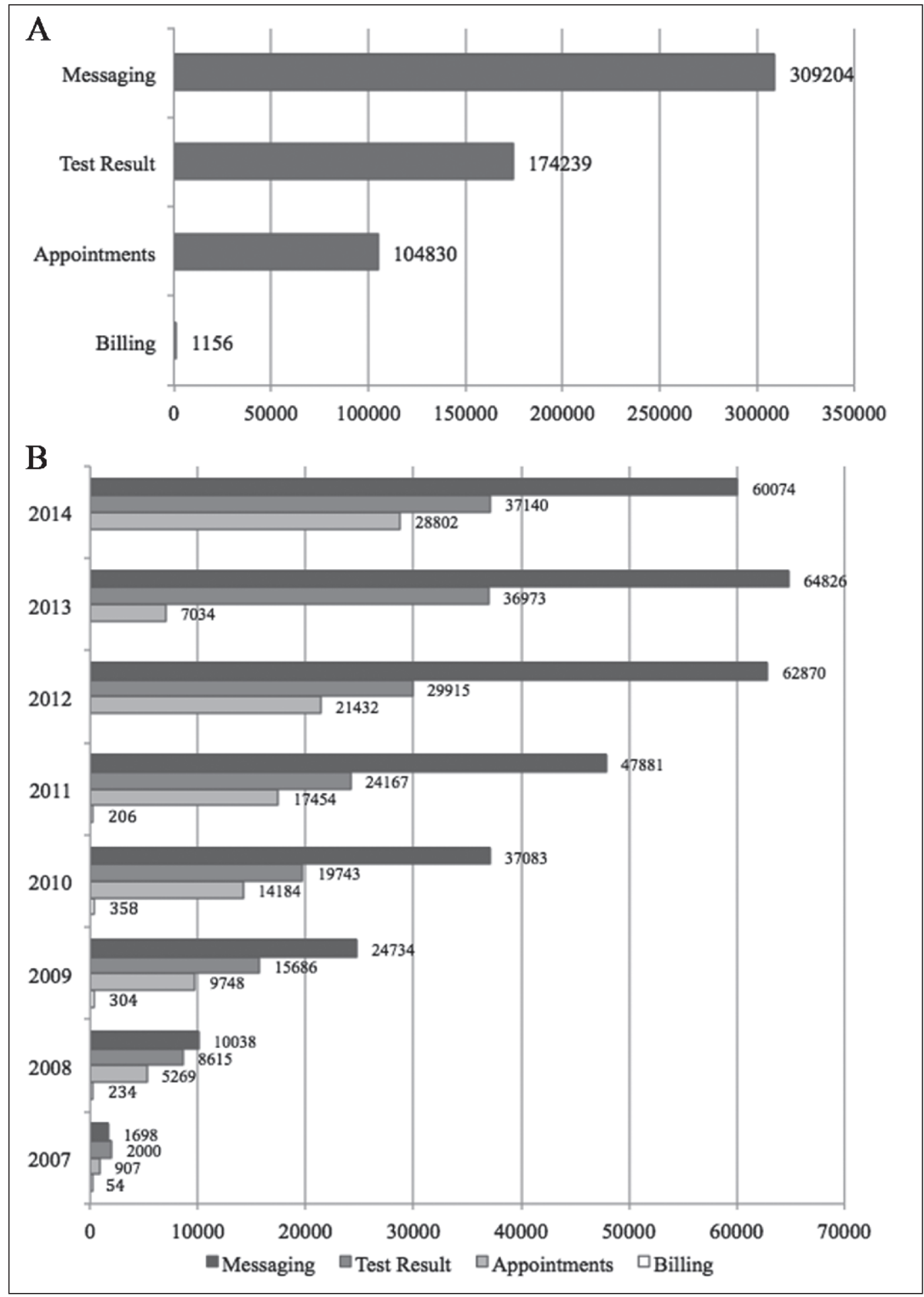

Fig. 3 A) Total MHAV function access, and B) MHAV function access by year 


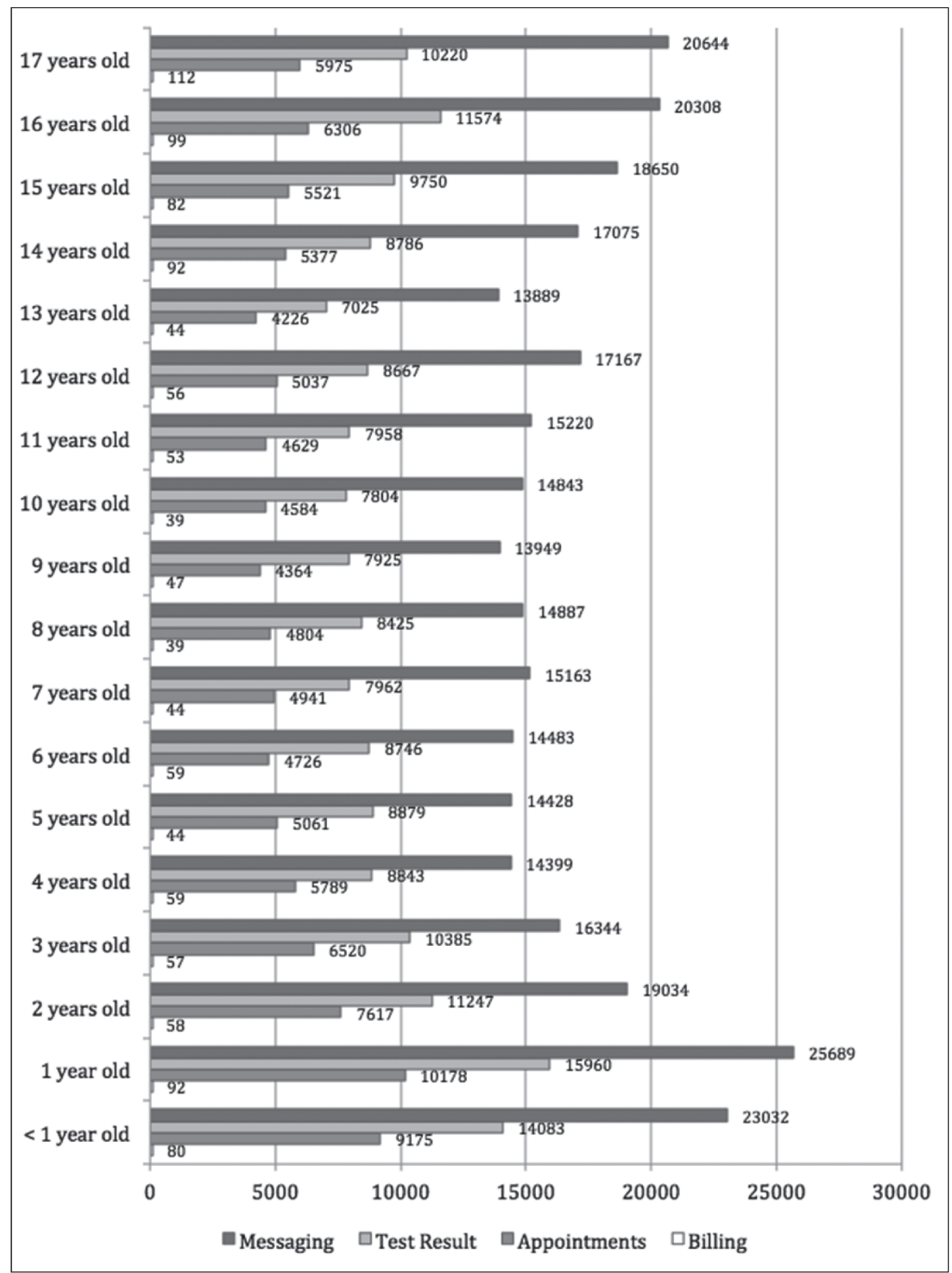

Fig. 4 Number of successful logins with messaging, test result, appointment, and billing function access by patient age 


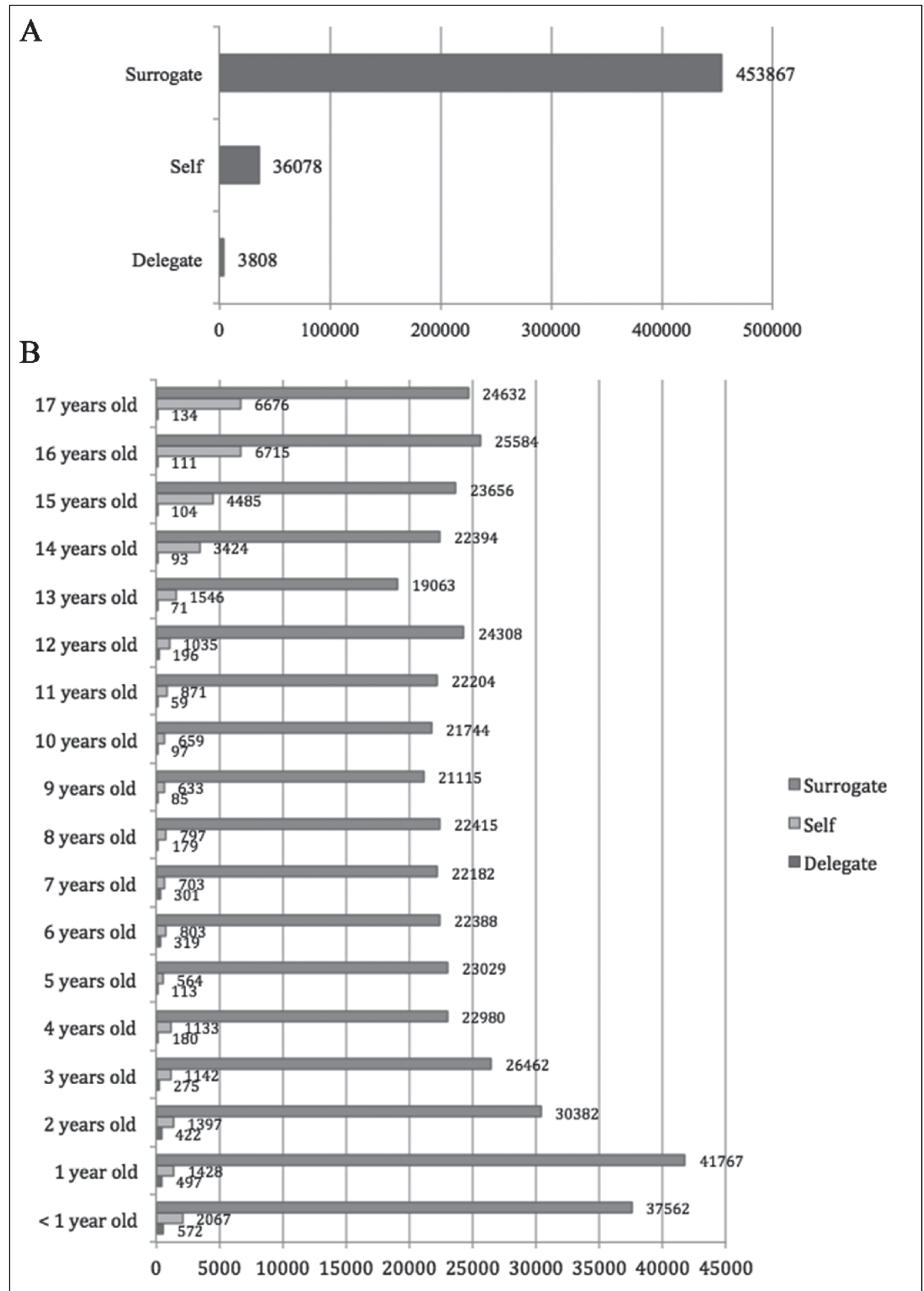

Fig. 5 A) Total login statistic by role and B) Login statistics by role and age 
Table 1 Demographic characteristics of the pediatric patients with associated MHAV accounts. Gender and race are represented as number of occurrences per category and percentage of population belonging to that category. Mean age is represented as the mean and interquartile range.

\begin{tabular}{|c|c|c|c|c|}
\hline & $\begin{array}{l}\text { Total } \\
(\mathrm{N}=17,128)\end{array}$ & $\begin{array}{l}\text { Self Accounts } \\
(N=2,720)\end{array}$ & $\begin{array}{l}\text { Surrogate Accounts } \\
(\mathrm{N}=16,036)\end{array}$ & $\begin{array}{l}\text { Delegate Accounts } \\
(\mathrm{N}=378)\end{array}$ \\
\hline \multicolumn{5}{|l|}{ Sex } \\
\hline Male & $8,793(51.3 \%)$ & $1,321(48.6 \%)$ & $8,283(51.7 \%)$ & $202(53.4 \%)$ \\
\hline Female & $8,334(48.7 \%)$ & 1,399 (51.4\%) & $7,752(48.3 \%)$ & $176(46.6 \%)$ \\
\hline Unknown & $1(<0.1 \%)$ & 0 & $1(<0.1 \%)$ & 0 \\
\hline \multicolumn{5}{|l|}{ Race } \\
\hline Asian & $632(3.7 \%)$ & $87(3.2 \%)$ & $615(3.8 \%)$ & $26(6.9 \%)$ \\
\hline African-American & 1,395 (8.1\%) & $232(8.5 \%)$ & $1,295(8.1 \%)$ & $23(6.1 \%)$ \\
\hline American Indian & $28(0.2 \%)$ & $5(0.2 \%)$ & $26(0.2 \%)$ & $1(0.3 \%)$ \\
\hline Caucasian & $13,725(80.1 \%)$ & $2,185(80.8 \%)$ & $12,871(80.3 \%)$ & $314(83.1 \%)$ \\
\hline Pacific Islander & $10(<0.1 \%)$ & $2(<0.1 \%)$ & $10(<0.1 \%)$ & 0 \\
\hline Unknown & $1,338(7.8 \%)$ & $209(7.7 \%)$ & $1,219(7.6 \%)$ & $14(3.7 \%)$ \\
\hline \multicolumn{5}{|c|}{ Age at MHAV enrollment } \\
\hline & & $<13$ years of age & & \\
\hline Mean (range) & & $5(0,12.9)$ & & \\
\hline \multirow[t]{2}{*}{ Median } & & 5 & & \\
\hline & & $\geq 13$ years of age & & \\
\hline Mean (range) & $7.9(0,17.9)$ & $15.1(13,17.9)$ & $7.5(0,17.9)$ & $5.3(0,17.9)$ \\
\hline Median & 8.0 & 15 & 7.3 & 3.3 \\
\hline
\end{tabular}




\section{References}

1. Archer N, Fevrier-Thomas U, Lokker C, McKibbon KA, Straus SE. Personal health records: a scoping review. AMIA 2011; 18(4): 515-22.

2. HealthIT.gov: What is a patient portal? [Internet]. 2014 Available from: healthit.gov.

3. Tang PC, Ash JS, Bates DW, Overhage JM, Sands DZ. Personal Health Records: Definitions, Benefits, and Strategies for Overcoming Barriers to Adoption. AMIA 2006; 13(2): 121-6.

4. Weingart SN, Rind D, Tofias Z, Sands DZ. Who uses the patient internet portal? The PatientSite experience. AMIA 2006; 13(1): 91-5.

5. Nazi KM, Woods SS, Woods SS. MyHealtheVet PHR: a description of users and patient portal use. AMIA Annu Symp Proc 2008; 1182.

6. Kaelber D, Pan EC. The value of personal health record (PHR) systems. AMIA Annu Symp Proc 2008; 343-7.

7. Detmer D, Bloomrosen M, Raymond B, Tang P. Integrated personal health records: transformative tools for consumer-centric care. BMC Med Inform Decis Mak. BioMed Central Ltd 2008; 8(1): 45-14.

8. Ralston JD, Hereford J, Carrell D. Use and satisfaction of a patient Web portal with a shared medical record between patients and providers. AMIA Annu Symp Proc 2006; 1070-1.

9. Goldzweig CL, Towfigh AA, Paige NM, Orshansky G, Haggstrom DA, Beroes JM, et al. Systematic Review: Secure Messaging Between Providers and Patients, and Patients' Access to Their Own Medical Record: Evidence on Health Outcomes, Satisfaction, Efficiency and Attitudes. Washington (DC): Department of Veterans Affairs (US) 2012 Jul; 1-63.

10.Davis SE, Osborn CY, Kripalani S, Goggins KM, Jackson GP. Health Literacy, Education Levels, and Patient Portal Usage During Hospitalizations. AMIA Annu Symp Proc 2015; 1-10.

11. Ross SE, Moore LA, Earnest MA, Wittevrongel L, Lin C-T. Providing a Web-based Online Medical Record with Electronic Communication Capabilities to Patients With Congestive Heart Failure: Randomized Trial. J Med Internet Res 2004; 6(2): e12.

12.Lin C-T, Wittevrongel L, Moore L, Beaty BL, Ross SE. An Internet-Based Patient-Provider Communication System: Randomized Controlled Trial. J Med Internet Res 2005; 7(4): e47.

13. Krist AH, Woolf SH, Rothemich SF, Johnson RE, Peele JE, Cunningham TD, et al. Interactive Preventive Health Record to Enhance Delivery of Recommended Care: A Randomized Trial. The Annals of Family Medicine 2012; 10(4): 312-9.

14. Wright A, Poon EG, Wald J, Feblowitz J, Pang JE, Schnipper JL, et al. Randomized Controlled Trial of Health Maintenance Reminders Provided Directly to Patients Through an Electronic PHR. J GEN INTERN MED. Springer-Verlag 2011; 27(1): 85-92.

15. Green BB. Effectiveness of Home Blood Pressure Monitoring, Web Communication, and Pharmacist Care on Hypertension Control. JAMA 2008; 299(24): 2857-20.

16. Ralston JD, Hirsch IB, Hoath J, Mullen M, Cheadle A, Goldberg HI. Web-Based Collaborative Care for Type 2 Diabetes: A pilot randomized trial. Diabetes Care 2009; 32(2): 234-9.

17. Tang PC, Overhage JM, Chan AS, Brown NL, Aghighi B, Entwistle MP, et al. Online disease management of diabetes: Engaging and Motivating Patients Online With Enhanced Resources-Diabetes (EMPOWER-D), a randomized controlled trial. AMIA 2013; 20(3): 526-34.

18. Simon GE, Ralston JD, Savarino J, Pabiniak C, Wentzel C, Operskalski BH. Randomized Trial of Depression Follow-Up Care by Online Messaging. J GEN INTERN MED 2011; 26(7): 698-704.

19. Goldzweig CL, Orshansky G, Paige NM, Towfigh AA, Haggstrom DA, Miake-Lye I, et al. Electronic Patient Portals: Evidence on Health Outcomes, Satisfaction, Efficiency, and Attitudes. Ann Intern Med. American College of Physicians 2013; 159(10): 677-25.

20.de Lusignan S, Mold F, Sheikh A, Majeed A, Wyatt JC, Quinn T, et al. Patients` online access to their electronic health records and linked online services: a systematic interpretative review. BMJ Open 2014; 4(9): e006021-11.

21. Thompson LA, Novak M, Schentrup AM. Adolescent Health and the Electronic Health Record: Can it be a social media tool for quality adolescent care? Adolesc Med State Art Rev 2014; 25(3): 698-710.

22. Bourgeois FC, Taylor PL, Emans SJ, Nigrin DJ, Mandl KD. Whose personal control? Creating private, personally controlled health records for pediatric and adolescent patients. AMIA 2008; 15(6): 737-743.

23. Bush RA, Connelly CD, Fuller M, Pérez A. Implementation of the Integrated Electronic Patient Portal in the Pediatric Population: A Systematic Review. Telemedicine and e-Health. 2015 Aug 10; $150810084832003-9$.

24. Ahlers-Schmidt CR, Nguyen M. Parent intention to use a patient portal as related to their children following a facilitated demonstration. Telemed J E Health 2013; 19(12): 979-981.

25. Bergman DA, Brown NL, Wilson S. Teen Use of a Patient Portal: A Qualitative Study of Parent and Teen Attitudes. Perspectives in Health Information Management / AHIMA, American Health Information Management Association 2008; 5: 13. 
26. Britto MT, Jimison HB, Munafo JK, Wissman J, Rogers ML, Hersh W. Usability Testing Finds Problems for Novice Users of Pediatric Portals. Journal of the American Medical Informatics Association 2009; 16(5): 660-669.

27. Britto MT, Hesse EA, Kamdar OJ, Munafo JK. Parents' perceptions of a patient portal for managing their child's chronic illness. The Journal of pediatrics 2013; 163(1): 280-281.e281-282.

28. Byczkowski TL, Munafo JK, Britto MT. Family perceptions of the usability and value of chronic disease web-based patient portals. Health informatics journal. 2014; 20(2): 151-162.

29. Fiks AG, Mayne S, Karavite DJ, DeBartolo E, Grundmeier RW. A shared e-decision support portal for pediatric asthma. The Journal of ambulatory care management 2014; 37(2): 120-126.

30. Thompson LA, Martinko T, Budd P, Mercado R, Schentrup AM. Meaningful Use of a Confidential Adolescent Patient Portal. Journal of Adolescent Health 2016; 58(2): 134-140.

31. Hannan A. Providing patients online access to their primary care computerised medical records: a case study of sharing and caring. Inform Prim Care 2010; 18(1): 41-49.

32. Ketterer T, West DW, Sanders VP, Hossain J, Kondo MC, Sharif I. Correlates of patient portal enrollment and activation in primary care pediatrics. Academic pediatrics 2013; 13(3): 264-271.

33. Shaw RJ, Ferranti J. Patient-provider internet portals - patient outcomes and use. Computers, informatics, nursing 2011; 29(12): 714-718; quiz 719-720.

34. Tom JO, Mangione-Smith R, Solomon C, Grossman DC. Integrated personal health record use: association with parent-reported care experiences. Pediatrics 2012; 130(1): e183-190.

35. Byczkowski TL, Munafo JK, Britto MT. Variation in use of Internet-based patient portals by parents of children with chronic disease. Archives of pediatrics \& adolescent medicine 2011; 165(5): 405-411.

36. Patient Volumes | Children's Hospital Vanderbilt [Internet]. Available from: http://www.childrenshospital. vanderbilt.org/guide.php?mid=3101

37. Osborn CY, Rosenbloom ST, Stenner SP, Anders S, Muse S, Johnson KB, et al. MyHealthAtVanderbilt: policies and procedures governing patient portal functionality. AMIA 2011; 18(Supplement 1): i18-i23.

38. Cronin RM, Davis SE, Shenson JA, Chen Q, Rosenbloom ST, Jackson GP. Growth of Secure Messaging Through a Patient Portal as a Form of Outpatient Interaction across Clinical Specialties. Appl Clin Inform 2015; 6(2): 288-304.

39. R: A language and environment for statistical computing. 2015. Vienna, Austria.

40. Crotty BH, Tamrat Y, Mostaghimi A, Safran C, Landon BE. Patient-to-physician messaging: volume nearly tripled as more patients joined system, but per capita rate plateaued. Health Aff (Millwood) 2014; 33(10): $1817-1822$.

41.Tennessee Population Demographics 2017, 2016 [Internet]. Available from: https://suburbanstats.org/ population/how-many-people-live-in-tennessee.

42.Kentucky Population Demographics 2017, 2016 [Internet]. Available from: https://suburbanstats.org/ population/how-many-people-live-in-kentucky.

43. Fiks AG, DuRivage N, Mayne SL, Finch S, Ross ME, Giacomini K, et al. Adoption of a Portal for the Primary Care Management of Pediatric Asthma: A Mixed-Methods Implementation Study. J Med Internet Res 2016; 18(6): e172

44. Wade-Vuturo AE, Mayberry LS, Osborn CY. Secure messaging and diabetes management: experiences and perspectives of patient portal users. AMIA 2013; 20(3): 519-525.

45. Palen TE, Ross C, Powers JD, Xu S. Association of Online Patient Access to Clinicians and Medical Records With Use of Clinical Services. JAMA 2012; 308(19): 2012-2018.

46.Women: Health Care Decision Makers [Internet]. Washington, DC; [cited 2016 Oct 9]. Available from: http://www.nationalpartnership.org/research-library/health-care/aca-enrollment-event-toolkit.pdf.

47. Masterman M, Cronin RM, Davis SA, Shenson JA, Jackson GP. Adoption of secure messaging in a patient portal across pediatric specialties. Proceedings of the AMIA 2016 Annual Symposium 2016, 1930-39.

48. Cronin RM, Fabbri D, Denny JC, Jackson GP. Automated Classification of Consumer Health Information Needs in Patient Portal Messages. AMIA Annu Symp Proc 2015; 2015: 1861-70.

49. Robinson JR, Valentine A, Carney C, Fabbri D, Jackson GP. Complexity of medical decision-making in care provided by surgeons through patient portals. Journal of Surgical Research 2017; 214: 93-101.

50. Robinson J, Davis SA, Cronin RM, Jackson GP. Use of a patient portal during hospital admissions to surgical services. Proceedings of the AMIA 2016 Annual Symposium 2016, 1967-76.

51. American Academy of P, American Academy of Family P, American College of P, Transitions Clinical Report Authoring G, Cooley WC, Sagerman PJ. Supporting the health care transition from adolescence to adulthood in the medical home. Pediatrics 2011; 128(1): 182-200. 